\title{
Depression: more than just serotonin
}

Serotonin and dopamine $\checkmark$ neurotransmitter systems in the brain are generally thought to function separately. In the presence of antidepressants that inhibit the reuptake of serotonin, however, their signals may become crossed. New research reveals that, in the presence of selective serotonin reuptake inhibitors (SSRIs), dopamine transporters may compensate to remove serotonin from the synapse. ${ }^{1}$

The corpus striatum is known to affect motor coordination, motivation and rewardbased learning. This area of the brain is densely packed with dopamine innervation, and it also has a moderate amount of serotonin innervation. The proximity of dopaminergic and serotoninergic neurons naturally facilitates interactions between them. ${ }^{2}$

Under normal circumstances, serotonin transporters remove serotonin from the synapse; SSRIs block these transporters and thus increase the concentration of serotonin.
However, dopamine transporters are known to have a low affinity for serotonin and may therefore act as a substitute for serotonin transporters.

In a recent study, Zhou and colleagues ${ }^{1}$ demonstrated in mice that, in the presence of the SSRI fluoxetine or an excess of extracellular serotonin, dopamine nerve terminals will take up serotonin from the synapse. These neurons, which normally release only dopamine, will then release both dopamine and serotonin when they next fire. The authors suggest that this "hijacking" of dopamine transporters by serotonin may explain why it takes a few days for the effects of SSRI therapy to appear.

Serotonin is thought to play a crucial role in the pathophysiology of depression. The clinical effect of dopamine transporters in SSRI therapy is unclear, because the results described in the study by Zhou and associates do not touch upon whether dopamine and serotonin signalling interac- tions affect behaviour. However, they do point to dopamine transporters as an intriguing target of future study, the

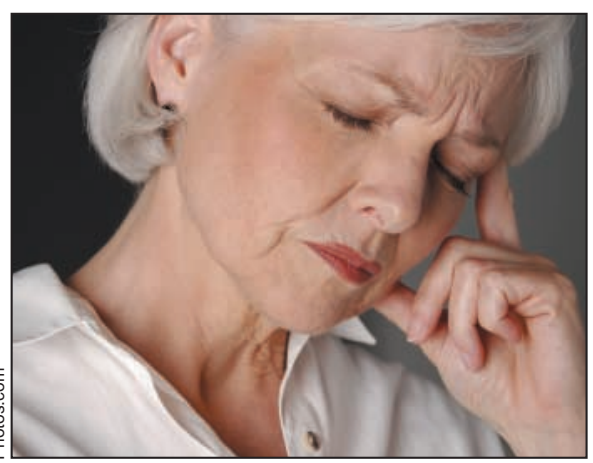

results of which may help in the design of new antidepressants that block the ability of dopamine receptors to uptake serotonin. - David Secko

\section{References}

1. Zhou FM, Liang Y, Salas R, Zhang L, De Biasi M, Dani JA, et al. Corelease of dopamine and serotonin from striatal dopamine terminals. Neuron 2005;46(1):65-74.

2. D'Aquila PS, Collu M, Gessa GL, Serra G. The role of dopamine in the mechanism of action of antidepressant drugs. Eur 7 Pharmacol 2000;405:365-73.

\section{A drug target for focal segmental glomerulosclerosis}

$\mathrm{F}$ ocal segmental glomerulosclerosis (FSGS) is a syndrome for which there is no reliable treatment, in part because of a poor understanding of its pathophysiology. However, recent research has identified a potential target for therapy.

FSGS is characterized clini$\infty$ cally by varying degrees of proteinuria; $50 \%$ of patients progress to end-stage renal disease within 10 years. On the basis of research involving a large family with hereditary FSGS, Winn and colleagues have identified a shared genetic mutation that results in an increase in the amount of calcium transported into glomerular cells. ${ }^{1}$ Since ion channels are easily inhibited through drug therapy, the authors of the study suggest that the modified protein may be a good target for the treatment of chronic kidney disease. David Secko

\section{Reference}

1. Winn MP, Conlon PJ, Lynn KL, Farrington MK, Creazzo T, Hawkins $\mathrm{AF}$, et al. A mutation in the TRPC6 cation channel causes familial focal segmental glomerulosclerosis. Science Express; May 5, 2005.

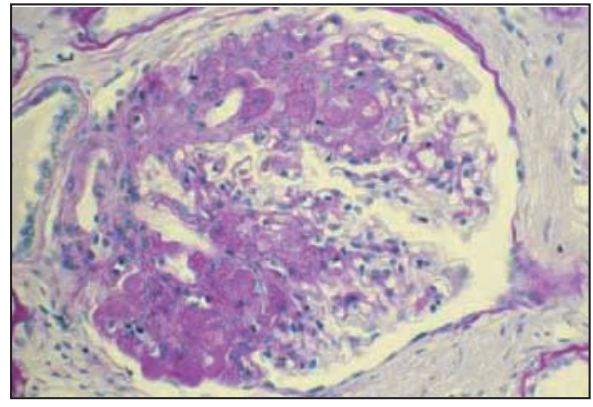

This PAS-stained section shows the perihilar location of sclerosis with hyalinosis and lipids vacuolation and an adhesion to Bowman's capsule, which are characteristic features of FSGS. Reproduced with the permission of Dr. J. Charles Jennette. 